\title{
Reliability of Attenuation Properties Recovery for Viscoelastic Media
}

\author{
Ekaterina Efimova, Vladimir Cheverda \\ The laboratory "Numerical methods of inversion of geophysical wavefields", A.A. Trofimuk Institute of Petroleum \\ Geology and Geophysics SB RAS. Novosibirsk, Russia \\ Email: EfimovaES@ipgg.sbras.ru
}

Received 2013

\begin{abstract}
The inverse problem of seismology for media with attenuation is considered in this paper. Generalized Standard Linear Solid is used to describe viscoelastic media. In the numerical solution certain parameterizations can be coupled, it means that true heterogeneity of the only one of parameters can be restored only as a perturbation of another. This is why important to investigate reliability of parameters recovery. By using method based on diffraction patterns it is possible to see whether the parameters are coupled. Singular value decomposition was used to study the possibility of recovering the parameters in practice. It was investigated the possibility of reconstructing of the density, impedances and attenuation properties. Coupling appears on the attenuation properties and impedances separately corresponding to the $\mathrm{P}$-wave and S-wave. It is also should be noted that coupling decreases with increasing frequency range and the condition number.
\end{abstract}

Keywords: Viscoelasticity; Seismic Attenuation; Inverse Theory; Wave Propagation; Singular Value Decomposition; Diffraction Patterns

\section{Introduction}

The main theme of the work is recovery of characteristics of viscoelastic media. For this purpose was considered numerical solution of two-dimensional inverse problem of seismology using the information recorded in the receivers located on the surface of the Earth. It is known that a numerical model of viscoelastic media describes the geological structures, in particular hydrocarbon reservoirs. And the attenuation properties depend on the composition of the fluid.

But there appears coupling of parameters of the medium in the numerical formulation of the problem that will be discussed in this article. Coupling of parameters means that the true heterogeneity of only one parameter would restore as a perturbation of other in the numerical solution. So if you use coupled parameters, your solution will be incorrect. Therefore, we need to find uncoupled set of parameters before developing and implementation of the algorithm.

Determination the possibility of uncoupled reconstruction of the parameters of a viscoelastic medium, such as density, elastic impedances and attenuation properties is the subject of this research.

This problem was considered in other papers, in particular, in [1], the problem was solved for the case of media with a velocity close to a constant value. There was also shown the inability simultaneous recovery of the velocity and attenuation properties in viscoelastic media without additional conditions [2].

\section{Numerical Description of Viscoelastic Media}

State equation provides the relationship between stresses and strains at the same time for ideal elastic media. But this is not true in viscoelastic media, which possess attenuation that caused by memory of the material. For such media stress state depends on all past states of strains and the state equation can be expressed with the use of the generalized Hooke's law. Media with attenuation mathematically can be described by the system of equations:

$$
\left\{\begin{array}{l}
\rho \partial \vec{u} / \partial t=\operatorname{div} \sigma+\vec{f} \\
\partial \varepsilon / \partial t=\left(\nabla \vec{u}+\nabla \vec{u}^{*}\right) / 2 \\
\sigma_{i j}(x, t)=\int_{-\infty}^{t} \partial \varepsilon_{k l}(x, \tau) / \partial \tau G_{i j k l}(x, t-\tau) d \tau .
\end{array}\right.
$$

\subsection{Generalized Standard Linear Solid}

Numerical resolution of such integral-differential system is very troublesome, so it is proposed to use Standard 
Linear Solid (SLS) - superposition of Maxwell medium and Kelvin-Voigt medium, to represent state equation in a differential form. The degree of attenuation(see in [3]) of a viscoelastic material is given by a Quality Factor $\mathrm{Q}$ (QF). Quality factor - the number of wavelengths a wave can propagate through a medium before its amplitude was decreased in $\exp (\pi)$ times.

It is known that a model that describes real geologic medium has constant quality factor in dependence of time frequency over the frequency range [4]. As one can see SLS does not satisfy this condition, in contrast to the further considered Generalized Standard Linear Solid (GSLS) [5] - a combination of several SLSs. Hooke's law in GSLS rewrites in the form of equations:

$$
\begin{aligned}
& \sigma=\sum_{j=1}^{L} \sigma_{j}, \\
& \sigma_{l}+\tau_{\sigma l} \partial \sigma_{l} / \partial t=M_{R}\left(\varepsilon+\tau_{\varepsilon l} \partial \varepsilon / \partial t\right),
\end{aligned}
$$

where $\mathrm{L}-$ is a number of SLS (further $\mathrm{L}=2$ );

$\tau_{\sigma l}, \tau_{\varepsilon l}$ - constants, that are called relaxation times of stresses and strains respectively; $M_{R}=\lambda+2 \mu$ for P-wave, $M_{R}=\mu$ for S-wave, - deformation modulus, where $\lambda, \mu$-Lame parameters.

Using GSLS model quality factor in the frequency domain can be rewritten as the equation (see [6]):

$$
Q=\left(1-L+\sum_{l=1}^{L} \frac{1+\tau_{\sigma l} \tau_{\varepsilon l} \omega^{2}}{1+\tau_{\sigma l}{ }^{2} \omega^{2}}\right) / \sum_{l=1}^{L} \frac{\left(\tau_{\varepsilon l}-\tau_{\sigma l}\right) \omega}{1+\tau_{\sigma l}{ }^{2} \omega^{2}},
$$

where the $\omega$ is a frequency .

To determine the relaxation times in [6] it is proposed to use $\tau$-method by introducing the parameters of attenuation - variables $\tau^{p}, \tau^{S} \quad \mathrm{P}, \mathrm{S}$ (corresponding to P-, $\mathrm{S}$-waves), that describe the level of attenuation in the medium. It should be noted that if we know the parameters of attenuation we have the only way to determine the quality factor:

$$
Q^{p} \approx \sum_{l=1}^{L} \frac{\tau^{p} \tau_{\sigma l} \omega^{2}}{1+\tau_{\sigma l}^{2} \omega^{2}}, Q^{s} \approx \sum_{l=1}^{L} \frac{\tau^{s} \tau_{\sigma l} \omega^{2}}{1+\tau_{\sigma l}^{2} \omega^{2}} .
$$

So in further considerations we will use the parameters of attenuation rather than Quality Factor because of several advantages of the $\tau$ - method that is listed in [6].

\subsection{Linearization}

After application of GSLS and the $\tau$ - method system of equations (1) can be regarded as a nonlinear operational equation: $B(\vec{m})=u^{o b s}$, where $\vec{m}$ - the parameters of the medium, $u^{\text {obs }}$ - observation data, $B$ is an operator from the space of models to the data space. It is suggested to use Newton's method: $D B\left(\vec{m}_{k}\right)<\vec{m}_{k+1}-\vec{m}_{k}>$ $=u^{\text {obs }}-B\left(\vec{m}_{k}\right)$, where $D B$ ia a - Frechet derivative of the operator $B, \vec{m}_{k}$ is model of the medium on the k-th step.

We mean that the parameters of the medium can be expressed as the sum of the constant components $\vec{m}_{0}$ and small perturbations $\delta \vec{m}: \vec{m}=\vec{m}_{0}+\delta \vec{m}$; then the total wave field will be presented as $\vec{u}=\vec{u}_{0}+\delta \vec{u}$, where $\vec{u}_{0}$ - propagating in a homogeneous medium wave, and $\delta \vec{u}$ - component generated by small perturbations of parameters. Small quantities of the second order are ignored in the linearization.

\section{Coupling Parameters}

Coupling between parameters means that in solution of the problem true heterogeneity of one parameter can be mistakenly identified as heterogeneity of another parameter [7]. The presence of coupling indicates wrong solution. Therefore, we will focus on methods identifying coupling sets of parameters.

\subsection{Diffraction Patterns}

To determine the coupling was studied the method of Tarantola [1986], based on the diffraction patterns [8]. Diffraction patterns show the amplitude of the wave, scattered from a point target area, as a function of the scattering angle. If diffraction patterns for different parameters has similar form it means coupling between parameters. A good choice of parameters will give diffraction patterns which are as different as possible.

The scattering diagrams were constructed for the sets of parameters:

1) Density, Lamé parameters, parameters of attenuation $\left\{\rho, \lambda, \mu, \tau^{p}, \tau^{s}\right\}$;

2) Density, velocities, parameters of attenuation $\left\{\rho, V^{p}, V^{s}, \tau^{p}, \tau^{s}\right\}$

3) Density, impedances, parameters of attenuation $\left\{\rho, I^{p}, I^{s}, \tau^{p}, \tau^{s}\right\}$.

The best results were obtained for parameterization $\left\{\rho, I^{p}, I^{s}, \tau^{p}, \tau^{s}\right\}$.

On Figure 1 are presented diffraction patterns for parameterization $\left\{\rho, I^{p}, I^{s}, \tau^{p}, \tau^{s}\right\}$ in the case of incident $\mathrm{P}$-wave and reflected $\mathrm{P}-, \mathrm{S}$-wawes. Diffraction patterns for the parameters of attenuation and impedances corresponding to $\mathrm{P}$-or S-wave are similar in shape, that indicates their coupling. Coupling may decrease when considering frequency range, as there is different dependence amplitude of the frequency for various parameters (Figure 1).

\subsection{Singular Value Decomposition}

To study the coupling and the possibility of recovery parameters is also used a method based on singular value decomposition (SVD) of a compact operator of the problem. Computation of singular value decomposition for an arbitrary environment is very complicated and expensive 
(in terms of computer resources) problem.

SVD analysis was performed for the environment model, which allows to determine the coupling between parameters (Figure 2). To construct the matrix representation of the operator target area was covered with a grid. Frequency range and positions of the receivers also were divided into finite segments. As the basis were used functions equal to one in the cell and zero outside. As a result, finite sums approach the integrals in the operator, that allows to get matrix approximation.

Because of the compactness of the operator it is proposed to consider the properties of solutions, using the truncated singular value decomposition [9]: it is considered r-solutions which are projections of the desired solution to the linear combination to the right singular vectors. The number of $r$ involved singular vectors controls conditionality of the problem and allows you to build a solution with acceptable accuracy.

To construct the singular numbers of the operator we have been considered different frequency ranges and different grid spacing. Convergence to zero of singular values (Figure 3) confirms the fact that the matrix is an approximation of a compact operator. With increasing of frequency range curves become flatter as you can see from the graphs, presented if the Figure 3. This indicates that in the case of a larger frequency range, we will have more vectors, to construct r-solutions, and thus increase the accuracy of the results.

To analyze coupling of parameterization we have constructed projection on the singular vectors that correspond to the largest singular numbers. Figures 4-6 show that the density couldn't be recovered, for other parameters only the amplitude of the gap on the boundary of the area with the perturbed parameter. Coupling occurs between the parameters $I^{p}, \tau^{p}$, and the parameters $I^{S}, \tau^{s}$.
Considering of larger frequency range allows minimize coupling. As well as increase of the condition number reduces coupling (Figures 4-6).

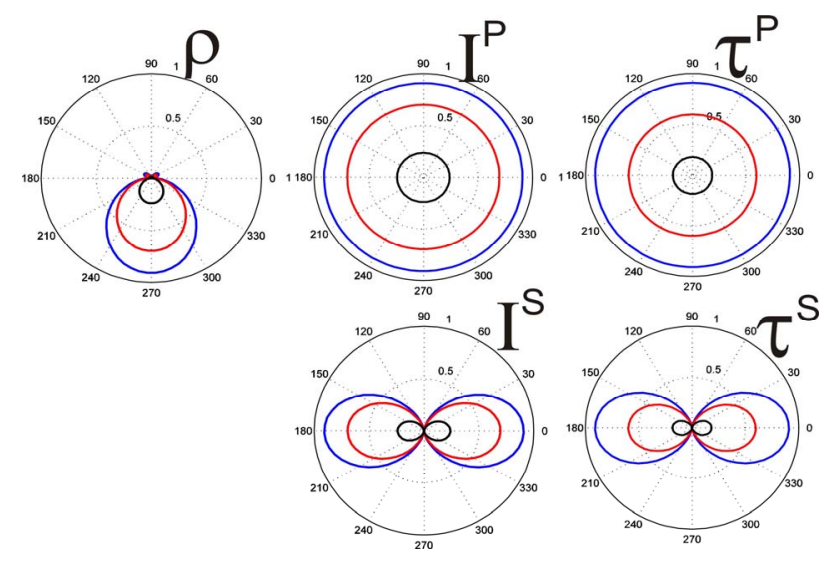

Figure 1. Diffraction patterns for the incident P-wave for frequencies $25 \mathrm{~Hz}, 15 \mathrm{~Hz}, 10 \mathrm{~Hz}$ (blue, red, black colors).

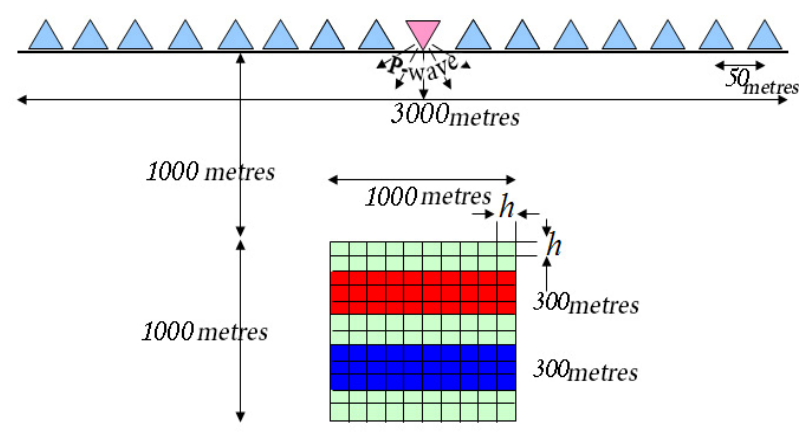

Figure 2. Mathematical model of the medium. Target area is a square. The red part has perturbation of the $S$-wave impedance, in blue - P-wave impedance; second case: S-wave attenuation - in red and P-wave attenuation - in blue.

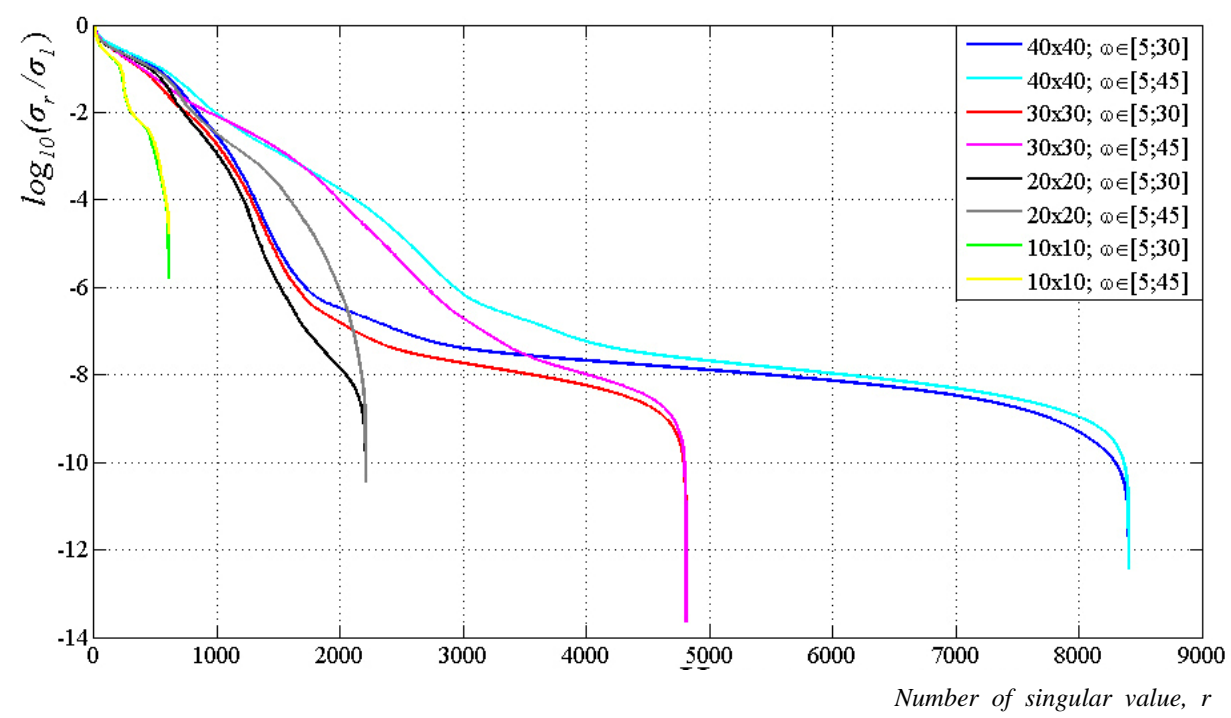

Figure 3. Singular values in the logarithmic scale for different grids(in metres) and frequency ranges (in $\mathrm{Hz}$ ). 


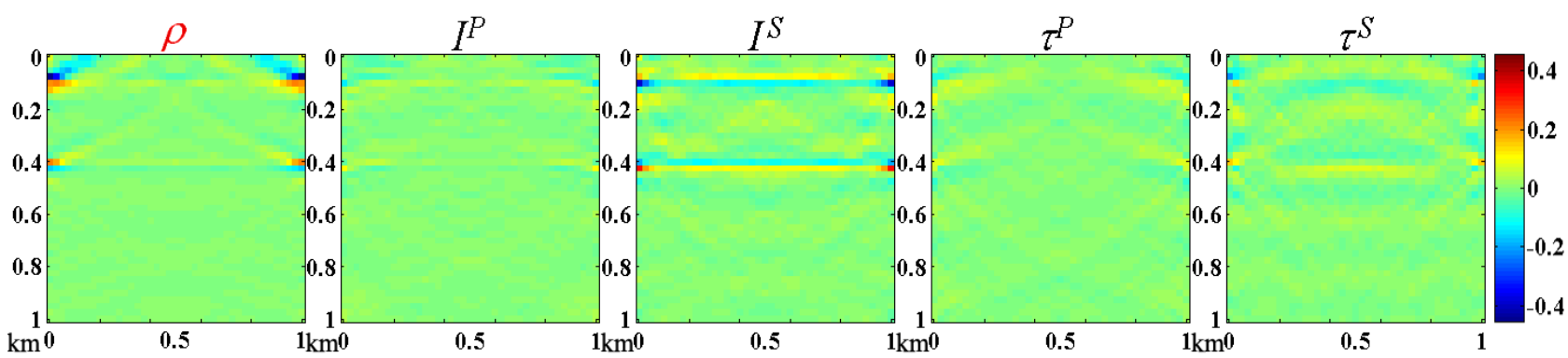

Figure 4. R-solutions for the model shown in Figure 2. In the upper layer - perturbation of dencity, the condition number is 10000 and a range of frequencies is $(5.45) \mathrm{Hz}$.

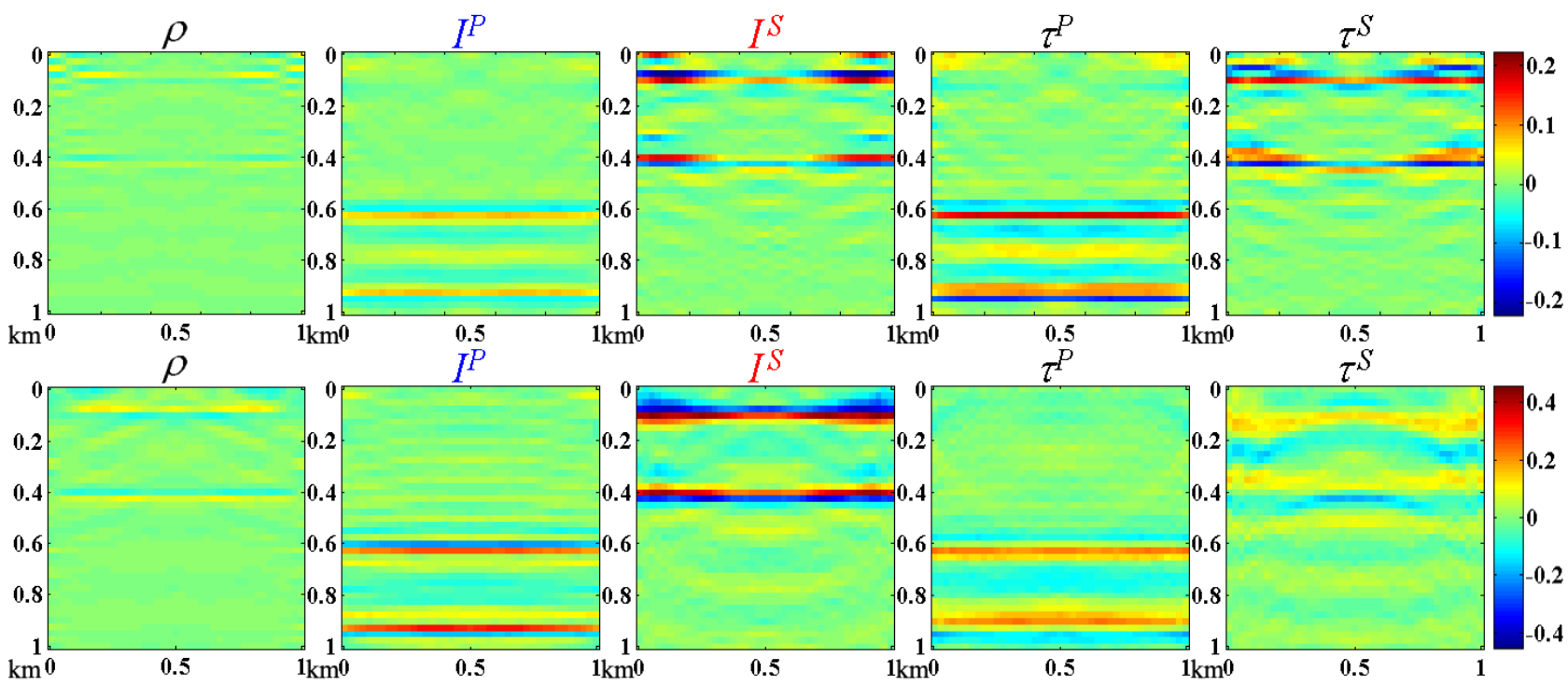

Figure 5. R-solutions for the model shown in Figure 2. In the upper layer - perturbation of S-wave impedance, in the lower of $\mathrm{P}$-wave impedance. In the first line of the condition number is 100 and the frequency range (5.30) $\mathrm{Hz}$ in the second - the condition number is 1000 and a frequency range is $(5.45) \mathrm{Hz}$.

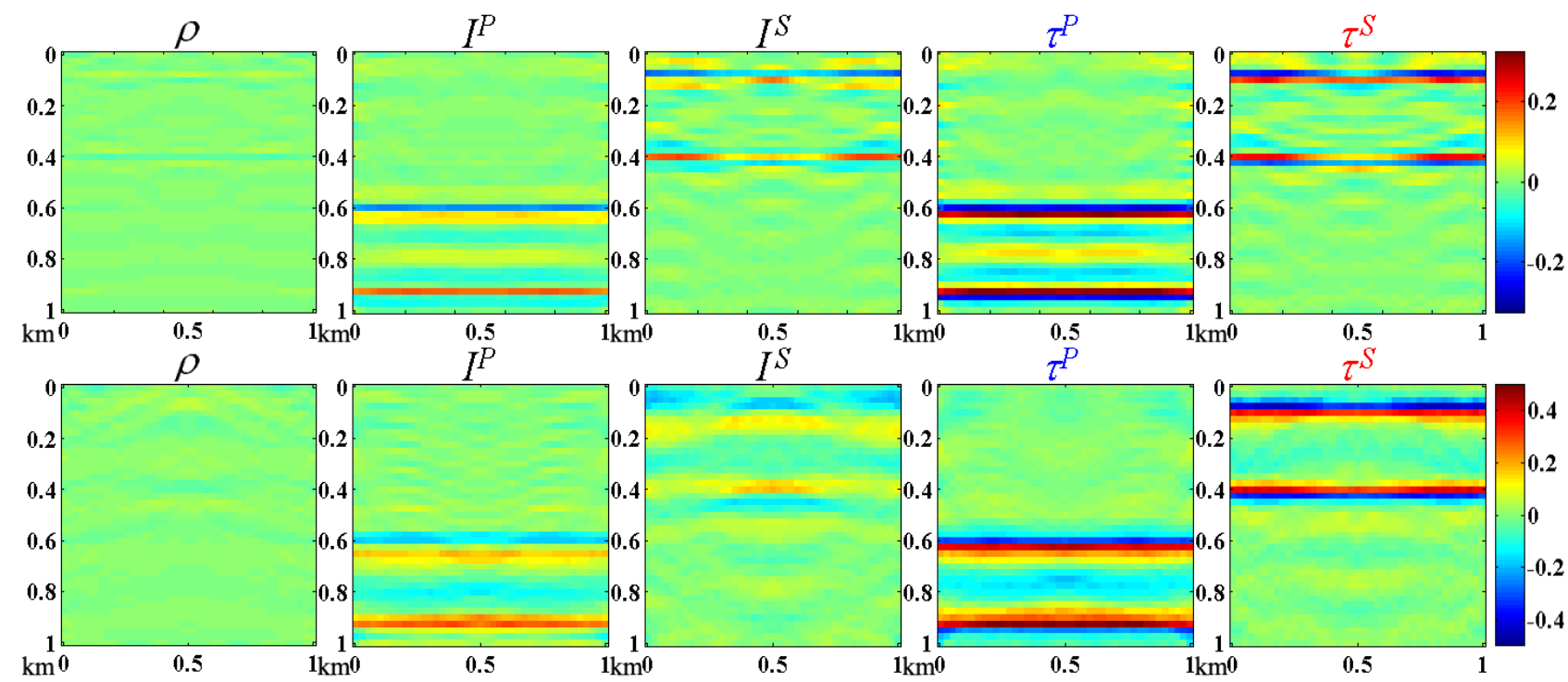

Figure 6. R-solutions for the model shown in Figure 2. In the upper layer - perturbation of S-wave attenuation, in the lower of $\mathrm{P}$-wave attenuation. In the first line of the condition number is 100 and the frequency range $(5.30) \mathrm{Hz}$ in the second - the condition number is 1000 and a frequency range is $(5.45) \mathrm{Hz}$. 


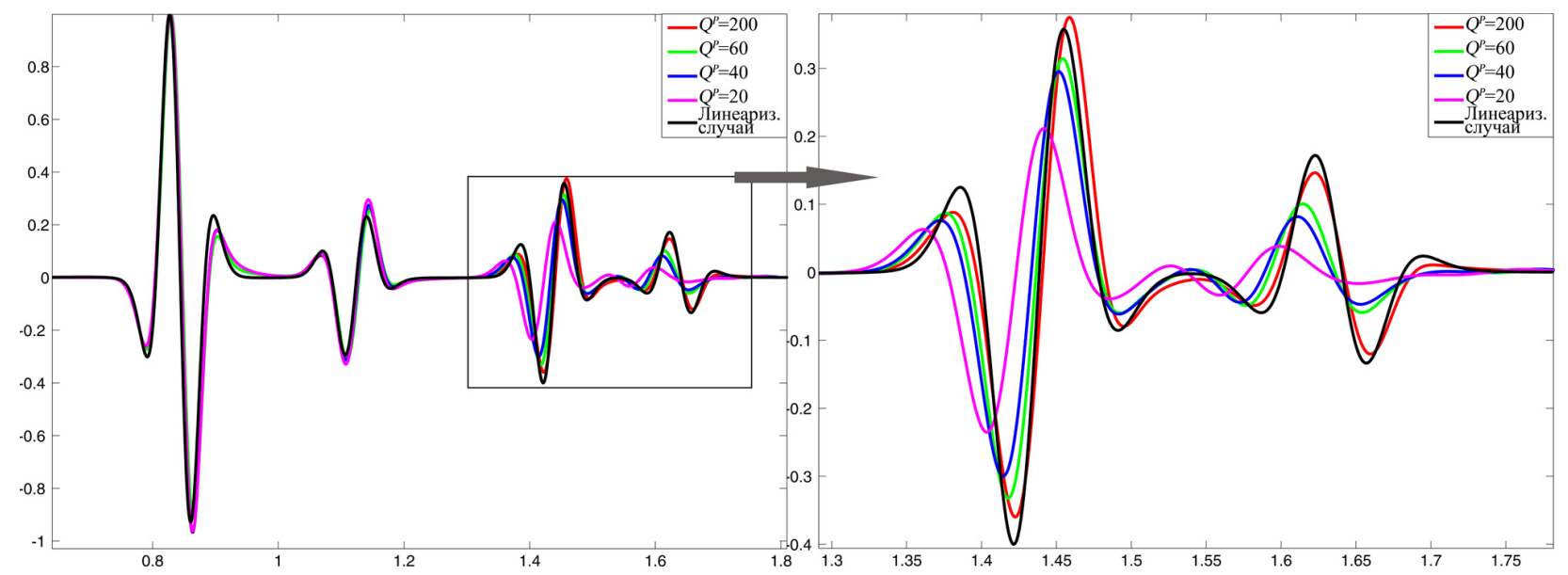

Figure 7. The scaled trace recorded in the first receiver in Fig. 2. Assumed the presence of $P$-wave attenuation: $Q=200,60$, 40, 20, and also in the linearized case. Right picture is zoomed rectangle from the left picture.

The presented in Figure 7 seismogram tracks shows that the graph for the linearized case with initial approximation acquisitions $\tau_{0}^{p}=0, \tau_{0}^{s}=0$ (the first approximation step of the viscoelastic medium) is most similar to the graph for the quality factor $Q=200$. This indicates that even a linear formulation gives good results.

\section{Conclusions}

To study the linearized operator of the dynamic theory of elasticity for the viscoelastic media were used methods based on diffraction patterns [8] and on the SVD-analysis [9]. The target area was chosen as a homogeneous medium with two areas, each has perturbation of only one parameter. This option provides an obvious determination of coupling of parameterization.

Coupling between the elastic impedances and the quality factors (corresponding to the $\mathrm{P}$ - and S-waves separately) was found.

By using diffraction patterns and singular value decomposition analysis was shown reducing coupling between parameters of viscoelastic medium in the case of increasing of the frequency range. Also was shown reducing coupling between parameters in the case of increasing of the condition number of the numerical problem.

It is concluded that the use of high-quality provides simultaneous reconstruction of impedances and quality factors. Study of an independent recovery of the parameters of viscoelastic medium with use of poor-quality data have planned for the future work.

\section{REFERENCES}

[1] J. M. Carcione, "Seismic Modeling in Viscoelastic Me- dia,” Geophysics, Vol. 58, No. 1, 1993, pp. 110-120. doi:10.1190/1.1443340

[2] W.A. Mulder and B. Hak "An Ambiguity in Attenuation Scattering Imaging,” Geophysical Journal International, Vol. 178, 2009, pp. 1614-1624. doi:10.1111/j.1365-246X.2009.04253.x

[3] S. Asvadurov, L. Knizhnerman and J. Pabon, "Finite-Difference Modeling of Viscoelastic Materials with Quality Factors of Arbitrary Magnitude,” Geophysics, Vol. 69, No. 3, 2004, pp. 817-824.doi:10.1190/1.1759468

[4] W. I. Futterman, "Dispersive Body Waves," Journal of Geophysical Research, Vol. 67, No. 13, 1962. pp. 52795291. doi:10.1029/JZ067i013p05279

[5] H. -P. Liu, D. L. Anderson, H. Kanamori, "Velocity Dispersion due to Anelasticity; Implications for Seismology and Mantle Composition," Geophysical Journal of Royal Astronomical Society, Vol. 47, 1976, pp. 41-58. doi:10.1111/j.1365-246X.1976.tb01261.X

[6] J. O. Blanch, J. O. A. Robertsson and W. W. Symes, "Modeling of a Constant Q: Methodology and Algorithm for an Efficient and Optimally Inexpensive Viscoelastic Technique,” Geophysics, Vol. 60, No. 1, 1995, pp. 176-184. doi:10.1190/1.1443744

[7] F. Assous and F. Collino, "A Numerical Method for the Explanation of Sensitivity: The Case of the Identification of the 2D Stratified Elastic Medium," Inverse Problems, Vol. 6, No. 4, 1990, pp. 487-514. doi:10.1088/0266-5611/6/4/003

[8] A. Tarantola, "A Strategy for Nonlinear Elastic Inversion of Seismic Reflection Data,” Geophysics, Vol. 51, No. 10, 1986, pp. 1893-1903. doi:10.1190/1.1442046

[9] V. A. Cheverda, V.I. Kostin, "R-Pseudoinverses for Compact Operators in Hilbert Spaces: Existence and Stability,” Journal of Inverse and Ill-Posed Problems, Vol. 3, No. 2, 1995, pp. 131-148. doi:10.1515/jiip.1995.3.2.131 